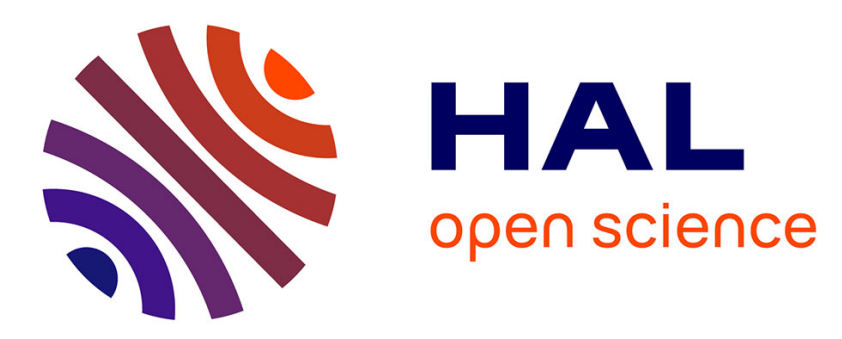

\title{
Surface plasma waves attenuation length at IR frequencies. Determination of the conduction electron scattering parameter at the metal-dielectric interface
}

J.C. Dudek

\section{- To cite this version:}

J.C. Dudek. Surface plasma waves attenuation length at IR frequencies. Determination of the conduction electron scattering parameter at the metal-dielectric interface. Revue de Physique Appliquée, 1984, 19 (4), pp.287-295. 10.1051/rphysap:01984001904028700 . jpa-00245195

\author{
HAL Id: jpa-00245195 \\ https://hal.science/jpa-00245195
}

Submitted on 1 Jan 1984

HAL is a multi-disciplinary open access archive for the deposit and dissemination of scientific research documents, whether they are published or not. The documents may come from teaching and research institutions in France or abroad, or from public or private research centers.
L'archive ouverte pluridisciplinaire HAL, est destinée au dépôt et à la diffusion de documents scientifiques de niveau recherche, publiés ou non, émanant des établissements d'enseignement et de recherche français ou étrangers, des laboratoires publics ou privés. 


\title{
REVUE DE PHYSIQUE APPLIQUÉE
}

Revue Phys. Appl. 19 (1984) 287-295

AVRIL 1984, PAGE 287

Classification

Physics Abstracts

$41.10-71.36-72.15 \mathrm{E}-73.25$

\section{Surface plasma waves attenuation length at IR frequencies. Determination of the conduction electron scattering parameter at the metal-dielectric interface}

\author{
J. C. Dudek
}

Faculté des Sciences d'Abidjan, 04-BP 422, Abidjan 04, Ivory Coast, and Laboratoire de Physique du Vide et des Composants Electroniques, CNAM, 292 rue Saint-Martin, 75141 Paris Cedex 03, France

(Reçu le 12 janvier 1983, révisé le 3 octobre, accepté le 11 janvier 1984)

\begin{abstract}
Résumé. - A température ambiante et pour une radiation dans le domaine du proche infra-rouge, l'impédance de surface $Z_{\mathrm{s}}$ des métaux usuels est très sensible à la valeur du paramètre de diffusion $p$ des électrons de conduction par l'interface métal-diélectrique. Nous montrons qu'à ces longueurs d'onde et pour une réflexion parfaitement diffuse $(p=0)$, la distance d'amortissement des ondes de plasma de surface est donnée avec une bonne approximation par une expression simplifiée faisant apparaître clairement l'influence de la conductivité, elle-même fonction de la température. Nous en déduisons une méthode expérimentale d'évaluation du paramètre $p$.
\end{abstract}

\begin{abstract}
At room temperature and for a radiation in the near infrared region the surface impedance of metals is very sensitive to the scattering parameter $p$ of the conduction electrons at the metal-dielectric interface. At these wavelengths and for a completely diffuse scattering, $(p=0)$, we show that a close estimate of the surface plasma waves (SPW) attenuation length is given by a simplified formula in which the influence of the temperature appears clearly via the dc bulk conductivity $\sigma$. We suggest then an experimental procedure for the determination of $p$.
\end{abstract}

\section{Introduction.}

The optical properties of metals are usually described by a local dielectric constant equal to the sum of two terms associated respectively with ions cores and conduction electrons excitations.

The latter is preponderant in the infrared and can be computed with a good approximation by using the classical Drude theory [1] at room temperature.

However, this theory is inadequate at low temperature and a more general and non-local approach. such as those used by Reuter and Sondheimer [2], is necessary to explain the " anomalous » experimental values of the surface impedance [3].

In addition to its non local form, the RS theory differs mainly from the Drude one by the fact that the scattering of the electrons by the surface is taken into account.
It is included in the boundary conditions by using the phenomenological parameter $p$ initially introduced by K. Fuchs in his theory of the size effect in electrical conductivity [4] and defined as the fraction of the conduction electrons specularly scattered.

To our knowledge, no complete theoretical attempt has been made to compute $p$; that is probably due to the great complexity of the phenomena involved and to the lack of reliable experimental results in the infrared domain at low temperature.

In numerous theoretical studies [5-8], $p$ has been taken equal to 1 because the equations are then far more simple than those implied by a partially or completely diffuse scattering.

However, that does not seem to be a realistic hypothesis if one considers the optical constants [9] or the surface plasma waves (SPW) attenuation 
lengths [10] obtained for the noble metals at room temperature, both measurements giving $p=0$. The same result has been deduced by Mc. Kay and Rayne [11] from absorptance measurements at low temperature.

On the other hand, in experiments on size effects in electrical conduction $[12,13] p$ has been found to have an intermediate value between 0 and 1 .

If a complete theory is ever conceived, it will presumably predict a dependence of $p$ on the incidence angle of the electrons [14] and on the roughness profile [13]. To a less extent, temperature and frequency dependence will probably also be found.

In this context, it is highly desirable to design accurate infrared experiments on well crystallized samples, the temperature of which being precisely controlled and the surface roughness characterized i.e. by its r.m.s. height.

Previous investigations $[10,15-17]$ have shown that SPW propagate over a few centimeters at room temperature in the $10 \mu \mathrm{m}$ region where surface scattering plays an important rôle.

Thus, the measurement of the attenuation length versus temperature could be used to test the RS theory and determine $p$; moreover, an eventual temperature dependance of $p$ could give information on the electron-surface phonon scattering.

In the following, after a short review of the main results of the classical electromagnetic theory of metals and the derivation of the classical dispersion relation of the SPW in section II, we will present in section 3 theoretical values of $Z_{\mathrm{s}}$ and of the attenuation length obtained for a $\mathrm{Cu}$-like metal in the framework of Reuter and Sondheimer anomalous skin effect theory.

The numerical calculations have been made using Dingle's series [18].

In section 4, an approximate dispersion relation deduced from Dingle's work and including the anomalous skin effect will lead us to a heuristic expression of the attenuation length valid in the 2-10 $\mu \mathrm{m}$ range.

As a consequence, an experimental method of determination of the scattering parameter will be proposed.

\section{Classical theory.}

If we consider a perfectly plane and smooth interface separating two adjacent semi-infinite media, one being a metal and the other a lossless dielectric of relative constant $\eta_{\mathrm{d}}$ (Fig. 1), an SPW will propagate [19] only if its wave vector $k_{x}$ satisfies the dispersion relation implied by the continuity of the tangential components of the fields $\boldsymbol{E}$ and $\mathscr{H}$ along the directions $\mathrm{O} x$ and $\mathrm{O} y$ respectively. Consequently, this dispersion relation will depend on the surface impedance of the metal defined by :

$$
Z_{\mathrm{s}}=\left.\frac{\mathcal{E}_{x}}{\mathscr{H}_{y}}\right|_{z=0}
$$

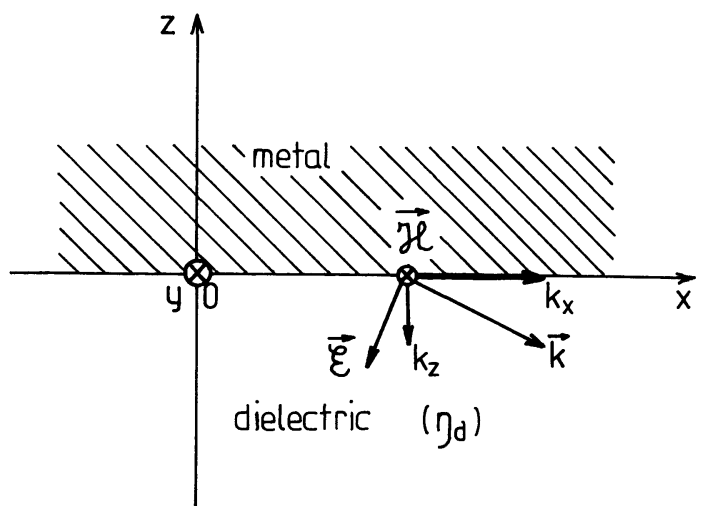

Fig. 1. - Metal-dielectric interface, coordinate system.

Maxwell's equations for the electric field in the dielectric :

$$
\nabla \times \mathcal{E}=-\mu_{0} \frac{\partial \mathfrak{H}}{\partial t}
$$

and :

$$
\nabla . \varepsilon=0
$$

( $\mu_{0}:$ vacuum permeability) can be written as :

$$
\begin{aligned}
\mathbf{k} \times \mathbf{E} & =\omega \mu_{0} \mathbf{H} \\
\mathbf{k . E} & =0
\end{aligned}
$$

where $\mathbf{E}$ and $\mathbf{H}$ are the complex amplitude vectors of the harmonic fields :

$$
\begin{aligned}
\boldsymbol{E} & =\mathbf{E} \exp [i(\mathbf{k . r}-\omega t)] \\
\boldsymbol{H} & =\mathbf{H} \exp [i(\mathbf{k . r}-\omega t)] .
\end{aligned}
$$

These expressions imply that the modulus of the wavevector in the dielectric is defined by the local relation :

$$
\mathbf{k}^{2}=\eta_{\mathrm{d}}\left(\frac{\omega}{c}\right)^{2}
$$

Equations (2) may be expressed from the components of the fields :

$$
\begin{aligned}
-k_{x} E_{z}+k_{z} E_{x} & =\omega \mu_{0} H_{y} \\
k_{x} E_{x}+k_{z} E_{z} & =0
\end{aligned}
$$

so that :

$$
\frac{\mathbf{k}^{2}}{k_{z}} E_{x}=\omega \mu_{0} H_{y}
$$

This relation which is true for any negative value of $z$ is also verified at $z=0$, and allows to express the continuity condition as follows :

$$
Z_{\mathrm{s}}=\omega \mu_{0} \frac{k_{z}}{\mathbf{k}^{2}}
$$


where $k_{z}=\left(k^{2}-k_{x}^{2}\right)^{1 / 2}$ with the condition $\operatorname{Im}\left\{k_{z}\right\}<0$, the SPW being linked to the interface.

From (3), a general continuity relation is obtained :

$$
Z_{\mathrm{s}}=\eta_{\mathrm{d}}^{1 / 2} Z_{0}\left(1-\frac{k_{x}^{2}}{\mathbf{k}^{2}}\right)^{1 / 2}
$$

in which $Z_{0}=\sqrt{\mu_{0} / \varepsilon_{0}}=376,730 \Omega$ is the free space impedance.

The generality of (4) is due to the fact that no particular hypothesis has been made about the spatial variation of the fields inside the metal.

Conversely, from the knowledge of $Z_{\mathrm{s}}$ the tangential wavevector can be computed by using the following dispersion relation :

$$
k_{x}=\eta_{\mathrm{d}}^{1 / 2} \frac{\omega}{c}\left[1-\eta_{\mathrm{d}}\left(\frac{Z_{\mathrm{s}}}{Z_{0}}\right)^{2}\right]^{1 / 2}
$$

with the condition $\operatorname{Im}\left\{k_{x}\right\}>0$ since the SPW is damped as it propagates along the $O \mathbf{x}$ axis in the positive direction.

Inside the metal, Ampere's theorem may be expressed as :

where :

$$
\nabla \times \mathfrak{U}=\mathbf{J}_{0}+\mathbf{J}_{\mathbf{i}}+\mathbf{J}_{\mathbf{c}}
$$

$\mathbf{J}_{0}=\varepsilon_{0} \frac{\partial \boldsymbol{E}}{\partial t}:$ displacement current in vacuum

$\mathbf{J}_{\mathbf{i}}=\frac{\partial \mathbf{P}_{\mathbf{i}}}{\partial t}=\varepsilon_{0} \alpha_{i} \frac{\partial \mathcal{E}}{\partial t}:$ current due to the polarization of the metallic ions.

In this paper, only metals in which the macroscopic polarizability $\alpha_{i}$ is a scalar will be considered. In addition, $\mathbf{J}_{0}$ and $\mathbf{J}_{i}$ will always be expressed by the preceding local relations whereas the current due to the conduction electrons $\mathbf{J}_{\mathbf{c}}$ will be eventually expressed by a nonlocal relation. That corresponds to the distinction commonly made between the purely local displacement component :

$$
\mathbf{J}_{\mathrm{d}}=\mathbf{J}_{0}+\mathbf{J}_{\mathrm{i}}=\eta_{\mathrm{r}} \varepsilon_{0} \frac{\partial \boldsymbol{\varepsilon}}{\partial t}
$$

where :

$$
\eta_{\mathrm{r}}=1+\alpha_{\mathrm{i}}
$$

is the total relative dielectric constant of the metal (ions plus vacuum) and $J_{\mathrm{c}}$ is the conduction component. In the infrared domain at frequencies therefore much lower than the plasma frequency of the metal, the condition $\nabla . \mathcal{E}=\mathbf{0}$ is fulfilled and only transverse E. M. waves $[20,29]$ have be considered.

The equation of propagation can be consequently written as :

$$
\nabla^{2} \boldsymbol{E}+\eta_{\mathrm{r}}\left(\frac{\omega}{c}\right)^{2} \boldsymbol{E}=-i \omega \mu_{0} \mathbf{J}_{\mathrm{c}}
$$

$\mathbf{J}_{c}$ is considered as local, that is proportional to $\boldsymbol{E}$ at any point when the following condition (page 341 of [2]) is satisfied :

$$
\frac{l}{\delta} \frac{1}{\left(1+\omega^{2} \tau^{2}\right)^{3 / 4}} \ll 1
$$

where $\delta=\left(2 / \sigma \omega \mu_{0}\right)^{1 / 2}$ is the classical skin depth and $l$ and $\tau$ are respectively the mean free path and the relaxation time of the conduction electrons.

Inequality (7) being fulfilled, Boltzmann's equation give the classical formula :

$$
\mathbf{J}_{\mathrm{c}} \# \frac{\boldsymbol{\sigma}}{1-i \omega \tau} \boldsymbol{\varepsilon}
$$

In (8), $\sigma=N e^{2} \tau / m^{*}$ is the bulk direct current conductivity; $N$ and $m^{*}$ are respectively the density and the effective mass of the conduction electrons.

$\tau$ depends on $l$ by the relation $: \tau=l / v_{\mathrm{F}}$, where $v_{\mathrm{F}}$ is the Fermi velocity.

Then, the propagation equation (6) may be rewriten under the usual following form :

$$
\nabla^{2} \boldsymbol{E}+\eta_{\mathrm{m}}\left(\frac{\omega}{c}\right)^{2} \boldsymbol{\varepsilon}=0
$$

with :

$$
\eta_{\mathrm{m}}=\alpha_{\mathrm{i}}+1-\frac{\omega_{\mathrm{p}}^{2}}{\omega^{2}}\left(1+\frac{i}{\omega \tau}\right)^{-1}
$$

in which expression ones recognizes the Drude dielectric constant of the metal.

$\omega_{p}=\left(N e^{2} / \varepsilon_{0} m^{*}\right)^{1 / 2}$ is the plasma angular frequency.

To a given value of $\omega$ corresponds therefore one wavevector $\mathbf{k}_{\mathrm{m}}$, the complex amplitude of which is defined by :

$$
\mathbf{k}_{\mathrm{m}}^{2}=\eta_{\mathrm{m}}\left(\frac{\omega}{c}\right)^{2}
$$

In this local approximation, relation (3) is valid and one obtain the surface impedance by the relation :

$$
Z_{\mathrm{s}}=\omega \mu_{0} \frac{k_{\mathrm{m} z}}{\mathbf{k}_{\mathrm{m}}^{2}}
$$

with :

$$
k_{\mathrm{mz}}=\left(\mathrm{k}_{\mathrm{m}}^{2}-k_{x}^{2}\right)^{1 / 2} .
$$

The inequality $\omega \ll \omega_{\text {p }}$ implied that :

$$
\eta_{\mathrm{m}} \#-\frac{\omega_{\mathrm{p}}^{2}}{\omega^{2}}\left(1+\frac{i}{\omega \tau}\right)^{-1}
$$

and :

$$
\left|\eta_{\mathrm{m}}\right| \gg 1
$$


As $\operatorname{Re}\left\{k_{x}\right\}$ is always of the order of $\omega / c$, we have :

$$
k_{\mathrm{m} z} \# \frac{\omega}{c} \eta_{\mathrm{m}}^{1 / 2}
$$

and $Z_{\mathrm{s}}$ is then given by the following simplified relation :

$$
Z_{\mathrm{s}} \# \frac{Z_{0}}{\left(\eta_{\mathrm{m}}\right)^{1 / 2}}
$$

This result indicates that for a given value of $\omega$, the surface impedance is practically independent of the tangential wavevector $k_{x}$ and is equal to the value rigorously obtained with a normally incident wave. The dispersion relation (5) can therefore be simplified as follows :

or :

$$
k_{x} \# \eta_{\mathrm{d}}^{1 / 2} \frac{\omega}{c}\left(1+\frac{\eta_{\mathrm{d}}}{2} \frac{\omega}{\omega_{p}^{2} \tau} i\right)
$$

$$
k_{x} \# \eta_{\mathrm{d}}^{1 / 2} \frac{\omega}{c}\left(1+\eta_{\mathrm{d}} \frac{\varepsilon_{0} \omega}{2 \sigma} i\right) .
$$

Thus, the classical attenuation length $L_{\mathrm{cl}}$ is given by :

$$
L_{\mathrm{cl}}=\frac{1}{J_{\mathrm{m} /}\left\{k_{x}\right\}} \# \frac{\lambda_{\mathrm{d}}^{2} Z_{\mathrm{d}}}{2 \pi^{2}} \sigma
$$

where :

$$
\text { d }-\quad 0 \quad \text { d } 0
$$

and $\lambda_{d}$ are respectively the volume impedance and wavelength in the dielectric.

\section{Nonlocal theory, anomalous skin effect.}

The classical theory becomes non valid when inequality (7) is not verified. Nonlocal effects are then present and the conduction current $\mathbf{J}_{c}$, at a given point, depends on the electric field values existing at the surrounding points. Therefore $\mathbf{J}_{\mathbf{c}}$ will also be strongly dependent on the electron scattering process at the interface. In nonlocal theories, $\mathbf{J}_{\mathbf{c}}$ will be expressed by pseudo convolution integrals having the following form [26] :

$$
\mathbf{J}_{c}(\mathbf{r}) \sim \iiint \mathrm{d}^{3} r^{\prime} \sigma\left(\mathbf{r}-\mathbf{r}^{\prime}\right) \boldsymbol{\varepsilon}^{\bullet}(\mathbf{r}) .
$$

From an experimental point of view, the measured value of $Z_{\mathrm{s}}$ at low temperature has a real part far greater than the theoretical estimate given by the classical theory [3] and the attenuation length is correspondingly much shorter than the value obtained from the expression (9) of $L_{\mathrm{cl}}$.

Moreover, the fields extend deeper in the metal than they do in classical conditions (anomalous skin effect) and their amplitude spatial variation is no longer given by an exponential law.
In the general case, for any intermediate value of $p$ between 0 and 1 , the exact computation of the spatial dependence of the fields is very difficult. However, if $p=1$ (specular scattering), the Boltzmann equation gives integral terms analogous to (10) in which the integration domain of each space coordinate extends from $-\infty$ to $+\infty[2,5]$.

Thus, one obtains true convolution integrals the space Fourier transforms of which, defined by :

$$
\mathbf{J}_{\mathbf{c}}(\mathbf{q})=\frac{1}{(2 \pi)^{3 / 2}} \iiint \mathrm{d}^{3} r \mathbf{J}_{\mathrm{c}}(\mathbf{r}) \exp (-i \mathbf{q} \cdot \mathbf{r})
$$

will give simply, after (10) :

$$
\mathbf{J}_{\mathbf{c}}(\mathbf{q}) \sim \sigma(\mathbf{q}) \boldsymbol{E}(\mathbf{q})
$$

$\sigma(q)$ is a nonlocal conductivity tensor depending on the wavevector $\mathbf{q}$ and implicitly on $\omega$, since in the above equations the wave is supposed to be monochromatic.

Then, one may compute the spectral distribution $\boldsymbol{E}(q)$ by using the propagation equation (6).

The surface impedance will be found from the definition :

$$
Z_{\mathrm{s}}=i \omega \mu_{0}\left(\frac{\partial \varepsilon_{x}}{\partial z}\right)_{z=Q^{+}}^{-1} \cdot \varepsilon_{x}(0)
$$

Space variation of the electric field in the metal is given by the inverse Fourier transform :

$$
\boldsymbol{E}(\mathbf{r})=\frac{1}{(2 \pi)^{3 / 2}} \iiint \mathrm{d}^{3} q \boldsymbol{\varepsilon}(\mathbf{q}) \exp (i \mathbf{q} \cdot \mathbf{r})
$$

If $p \neq 1$, a relation as (11) cannot be written and to our knowledge this problem has only been solved exactly in the two limit cases $p=1$ and 0 , by Reuter and Sondheimer [2] for a normally incident wave and by Kliewer, Fuchs and Keller [5, 22] for an obliquely incident wave.

A consequence of the latter theory is that one can neglect the variations of $Z_{\mathrm{s}}$ with the tangential wavevector $k_{x}$ if $\omega \ll \omega_{\mathrm{p}}$.

Thus, in the infrared domain, $Z_{\mathrm{s}}$ may be computed in the framework of Reuter and Sondheimer theory well adapted to numerical calculations by Dingle [23] who expanded the initial complicated integrals in series.

It should be noticed that the $k_{x}$ independence of $Z_{\mathrm{s}}$ had already been mentioned in the classical case treated in section 2 .

Expressed in MKSA units and for fields varying in time by an $\exp (-i \omega t)$ factor, the surface impedance given by the RS theory is :

$$
Z_{\mathrm{s}}=-\frac{i k_{0} l}{1-i \omega \tau} Z_{0} F_{p}(\xi)
$$

where :

$k_{0}=2 \pi / \lambda_{0}:$ free space wavevector, 
$Z_{0}:$ vacuum impedance,

$F_{p}:$ a function of the dimensionless complex parameter :

$$
\xi=-\frac{3}{2} i \frac{l^{2}}{\delta^{2}} \frac{1}{(1-i \omega \tau)^{3}}
$$

in which :

$$
\delta=\left(2 / \sigma \omega \mu_{0}\right)^{1 / 2}
$$

is the classical skin depth

If $p=0$ or $1, F_{p}(\xi)$ is expressed by Dingle's series :

- For diffuse scattering :

$$
\begin{aligned}
& \begin{aligned}
& F_{p=0}^{-1}(\xi) \approx 1.1547 \xi^{1 / 2}-0.2500 \xi+0.1540 \xi^{3 / 2}-0.1262 \xi^{2}+0.1188 \xi^{5 / 2}-0.1214 \xi^{3}+ \\
&+0.13075 \xi^{7 / 2}-0.148 \xi^{4}+0.1685 \xi^{9 / 2} \ldots
\end{aligned} \\
& \begin{aligned}
\text { if }|\xi|<0.8 \\
F_{p=0}^{-1}(\xi) \approx 1.157(\pi \xi)^{1 / 3}-\{0.1351 \ln (\pi \xi)+0.5330\}+0.0416(\pi \xi)^{-1 / 3}-0.0649(\pi \xi)^{-2 / 3}+ \\
+(\pi \xi)^{-1} \cdot\{-0.0164 \ln (\pi \xi)+0.0184\}+0.0660(\pi \xi)^{-4 / 3}-0.0307(\pi \xi)^{-5 / 3}
\end{aligned}
\end{aligned}
$$

if $|\xi|>0.8$.

- For specular scattering :

$F_{p=1}(\xi) \approx 0.8660 \xi^{-1 / 2}+0.1155 \xi^{1 / 2}-0.1667 \xi+0.2210 \xi^{3 / 2}-0.2901 \xi^{2}+$

$$
+0.3815 \xi^{5 / 2}-0.5040 \xi^{3}+0.6688 \xi^{7 / 2}-0.892 \xi^{4}+1.193 \xi^{9 / 2}-1.60 \xi^{5} \ldots
$$

if $|\xi|<0.8$

$$
\begin{aligned}
F_{p=1}(\xi) \approx & 0.7698(\pi \xi)^{-1 / 3}+0.6534(\pi \xi)^{-2 / 3}+(\pi \xi)^{-1}\{0,1318 \ln \{\xi\}+0.0852\}- \\
& -0.1325(\pi \xi)^{-4 / 3}+0.1983(\pi \xi)^{-5 / 3}+(\pi \xi)^{-2}\{0.0819 \ln (\pi \xi)-0.0788\}-0.1047(\pi \xi)^{-7 / 3}
\end{aligned}
$$

if $|\xi|>0.8$.

When computing the $\xi^{m}$ terms ( $m$ being eventually fractional and greater than one in absolute value), care must be taken to express the phase by $m \operatorname{Arg}(\xi)$ modulo $2 \pi$ and not modulo $\pi$ as sometimes occurs in subroutines of high level programming languages such as Fortran.

Curves of figures 2 and 3 represent the ratios of the real and imaginary parts of the copper surface impedance to their classical values.

To compute them in the isotropic approximation, one need only two intrinsic constants of the metal, for example :

$$
\frac{\sigma}{l}=\frac{N e^{2}}{m^{*} v_{\mathrm{F}}} \text { and } v_{\mathrm{F}}
$$

The datas used here for $\mathrm{Cu}$ are :

$$
\frac{\sigma}{l}=1.515 \times 10^{15} \Omega^{-1} \mathrm{~m}^{-2}
$$

and :

$$
\mathrm{v}_{\mathrm{F}}=1.59 \times 10^{6} \mathrm{~ms}^{-1}[3] .
$$

At room temperature the mean free path $l$ is about $0.03 \mu \mathrm{m}[13]$ and it may attain $8 \mu \mathrm{m}$ at liquid helium temperature [24].

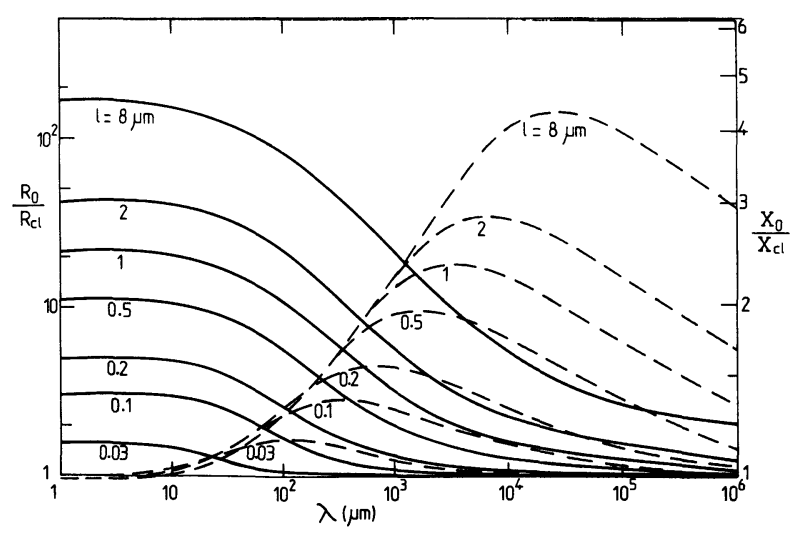

Fig. 2. - Surface impedance of copper when conduction electrons are diffusely scattered $(p=0)$. — ratio of the real part of $Z_{\mathrm{s}}$ to its classical value, - . - ratio of the imaginary part of $Z_{\mathrm{s}}$ to its classical value.

For $p=0$, Dingle's series present no significant discontinuity in the critical region corresponding to $|\xi| \approx 0.8$ where (13a) and (13b) overlap.

That is not the case for $p=1$ since the series (13c) in ascending powers of $\xi$ diverges strongly as $|\xi|$ reaches the critical value. 


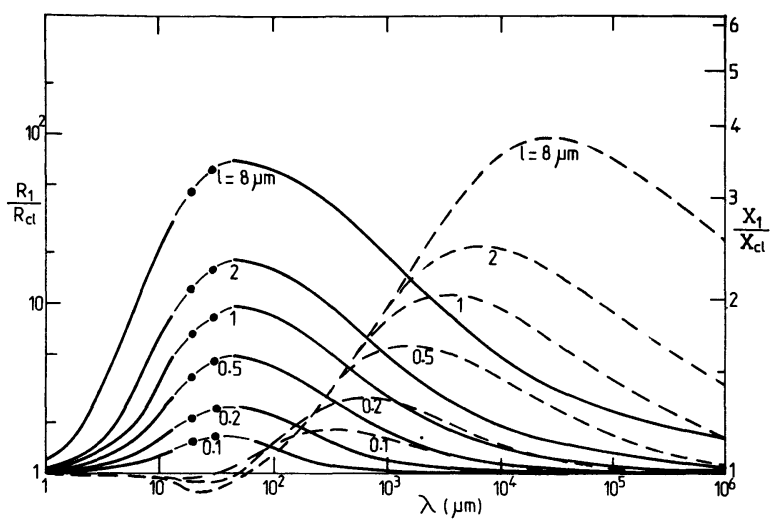

Fig. 3. - Surface impedance of copper when conduction electrons are specularly scattered $(p=1)$. — ratio of the real part of $Z_{\mathrm{s}}$ to its classical value, ... ratio of the imaginary part of $Z_{\mathrm{s}}$ to its classical value, $\multimap$ points obtained from direct integration in the critical domain $0.4 \lesssim|\xi| \lesssim 1.6$.
One thus obtains much too large values of $\operatorname{Re}\left\{Z_{\mathrm{s}}\right\}$ and it is necessary to compute the corresponding parts of the curves by direct numerical integration of $F_{p=1}(\xi)$ in expression (12).

The initial integral given by the RS theory is :

$$
F_{1}(\xi)=\frac{2}{\pi} \int_{0}^{\infty} \frac{\mathrm{d} t}{t^{2}+\xi \chi(t)}
$$

where

$$
\chi(t)=2 t^{-3}\left[\left(1+t^{2}\right) \operatorname{Arctg} t-t\right]
$$

As recommended by Reuter and Sondheimer, one has better to compute (14) using the convenient equivalent following expression :

$$
F_{1}(\xi)=I_{1}(\xi)-\xi I_{2}(\xi)
$$

with :

$$
I_{1}(\xi)=-\frac{2 i}{2 t_{0}+\xi \chi^{\prime}\left(t_{0}\right)}
$$

and :

$$
I_{2}(\xi)=2 \int_{0}^{1} \frac{\left(1-v^{2}\right) v^{3} \mathrm{~d} v}{\left\{1-\xi v^{3}\left[2 v+\left(1-v^{2}\right) \ln \left(\frac{1+v}{1-v}\right)\right]\right\}^{2}+\left[\pi \xi v^{3}\left(1-v^{2}\right)\right]^{2}}
$$

being the root of the characteristie equation :

$$
t^{2}+\xi \chi(t)=0
$$

and :

$$
\chi^{\prime}(t)=\frac{\mathrm{d} \chi}{\mathrm{d} t}=2 t^{-3}\left[3-\left(\frac{3}{t}+t\right) \operatorname{Arctg} t\right]
$$

Proceeding like that, one avoids computing (14) the integrand of which exhibits a quasi-singularity when the pole $t_{0}$ approaches to the real axis, that is when $\omega \tau$ becomes greater than unity.

Outside the critical domain approximately defined by $0.4<|\xi|<1.6$, direct integration and Dingle's series are in very good agreement, the coincidence being within $0.1 \%$.

Introducing the numerical values of $Z_{\mathrm{s}}$ in the dispersion relation (5) of section 1 , the attenuation length $L=1 / \operatorname{Jm}\left\{k_{x}\right\}$ may be calculated exactly. Figure 4 shows the variation of $L$ as a function of the mean free path $l$ for a $\mathrm{Cu}$-vacuum interface. At room temperature and $10 \mu \mathrm{m}$ wavelength, $L_{(p=0)}$ is significantly different from $L_{(p=1)}$ or $L_{\mathrm{cl}}$. That is due to the real part of $Z_{\mathrm{s}}$ (Fig. 2) which is far greater than what the classical or specular nonlocal theories give. It is noteworthy that at room temperature $(l \approx 0.03 \mu \mathrm{m})$ and $\lambda=10 \mu \mathrm{m}$, $|\xi|$ has the value 0.14 which, accordingly to inequality (7), should correspond to a "weak anomaly ».

The paradox mentioned by Dingle shows that for $p=0$, the classical results are not valid even at room temperature.

In the visible and near infrared, this phenomenon is partially hidden by the purely local displacement current which is no longer negligible compared with the conduction current.

Moreover, in most cases, the scattering parameter value lies probably between 0 and $1[25,12]$ and the effect is then less pronounced than if the electrons were diffusely scattered.

Figure 5 shows the SPW attenuation $A=\operatorname{Jm}\left\{k_{x}\right\}$ expressed by its ratio to the classical value.

The curves plotted for $p=0$ are easily distinguishable from those plotted for $p=1$ for the wavelengths shorter than about $100 \mu \mathrm{m}$. 


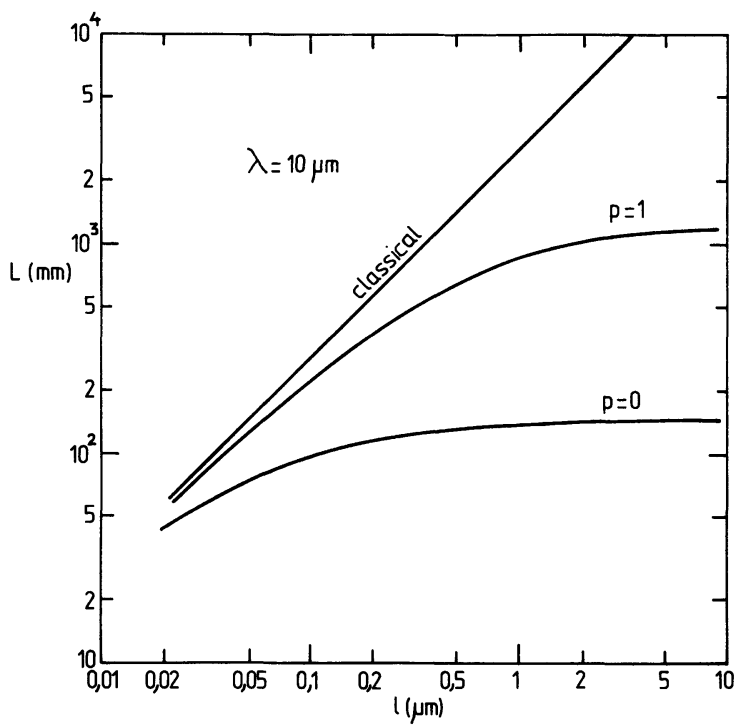

Fig. 4. - Exact theroretical attenuation length of a SPW propagating along a $\mathrm{Cu}$-vacuum interface at $10 \mu \mathrm{m}$ wavelength versus the mean free path $l$ of the conduction electrons.

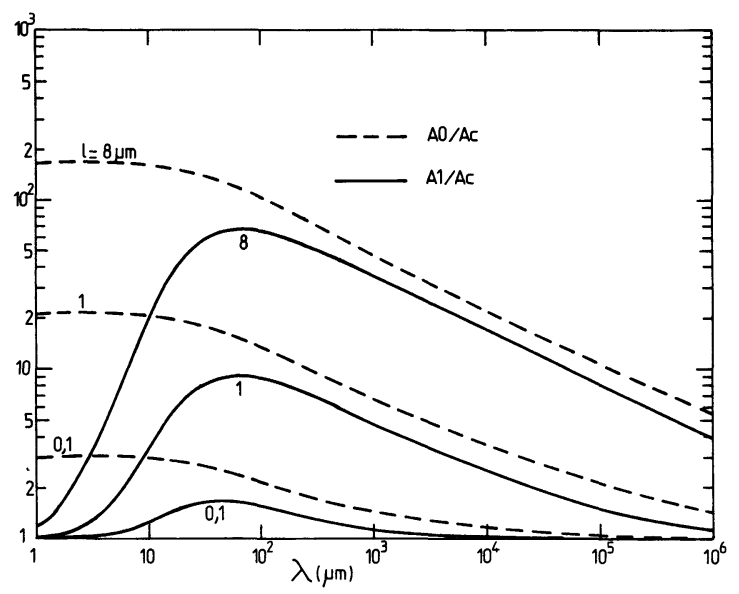

Fig. 5. - Ratio of the attenuation $A=\operatorname{Im}\left\{k_{x}\right\}$ of a SPW propagating along a $\mathrm{Cu}$-vacuum interface to its classical value $A_{\mathrm{C}}$ versus the wavelength $\lambda$... curves obtained for $p=0,-$ curves obtained for $p=1$.

On the other hand, when the electrons are specularly scattered, the anomaly remains very weak below a few $\mu \mathrm{m}$ and one may conclude that wavelengths around $10 \mu \mathrm{m}$ are particularly appropriate to the experimental investigation of these effects.

\section{Approximate dispersion relation, determination of $\boldsymbol{p}$.}

If $|\xi| \ll 1$ and $\omega \tau \gg 1$, that is if $\lambda$ is shorter than or equal to about $10 \mu \mathrm{m}$ at room temperature (that will be justified further), one can retain only the first two terms of (13a) and the first one of (13c) ([18] page 340 ).

$Z_{\mathrm{s}}$ can then be expressed by the following approxi- mate relations :

$$
\begin{aligned}
& Z_{\mathrm{s}}(p=1) \# Z_{0}\left(\frac{1}{2 \omega_{p} \tau}-\frac{\omega}{\omega_{p}} i\right) \\
& Z_{\mathrm{s}}(p=0) \# Z_{0}\left[\frac{1}{2 \omega_{p} \tau}\left(1+\frac{3}{8} \frac{l \omega_{p}}{\mathrm{c}}\right)-\frac{\omega}{\omega_{p}} i\right]
\end{aligned}
$$

where $Z_{0}$ is the free space impedance.

As in the above section, the fields will be supposed to vary with time by the factor $\exp (-i \omega t)$.

The surface impedance being expressed by $Z_{\mathrm{s}}=R+i X \quad(X<0)$ and since $\left|Z_{\mathrm{s}}\right| \ll Z_{0}$, the dispersion relation for the metal-vacuum interface may be written after (5) :

$$
k_{x} \# \frac{\omega}{c}\left(1-\frac{R X}{Z_{0}^{2}} i\right)
$$

or, from (15) :

$$
k_{x}(p=1) \# \frac{\omega}{c}\left(1+\frac{\omega}{2 \omega_{p}^{2} \tau} i\right)
$$

and :

$$
k_{x}(p=0) \# \frac{\omega}{c}\left[1+\frac{\omega}{2 \omega_{p}^{2} \tau}\left(1+\frac{3}{8} \frac{l \omega_{p}}{c}\right) i\right]
$$

(16a) is nothing else but the classical dispersion relation found in section 2 .

For copper, the additional term $\frac{3}{8} \frac{l \omega_{p}}{c}$ in is equal to about 0.6 at room temperature; therefore, it cannot be neglected and elucidates the feature of the attenuation curves obtained for $p=0$ (Fig. 5). For copper, the preceding approximate relations are valid inside a wavelength domain approximately defined by the two limiting values 2 and $10 \mu \mathrm{m}$. Under the lower limit the displacement current is not negligible compared to the conduction current and the validity of Dingle's series breaks down. Beyond the upper limit, the condition $|\xi| \ll 1$ is not satisfied.

$\omega \tau$ attains its smallest value 3.5 at room temperature for $\lambda=10 \mu \mathrm{m}$.

At lower temperatures or higher frequencies $\omega \tau$ is indeed much greater than unity and the modulus of $\xi$ may be expressed by :

$$
|\xi| \# \frac{3}{4} \frac{\sigma}{l} \frac{\mu_{0} v_{\mathrm{F}}^{3}}{\omega^{2}}
$$

Since $\sigma / l$ is a constant, $|\xi|$ depends only on $\omega$ and therefore, the upper limit of the preceding wavelength domain defined by the condition $|\xi| \ll 1$ does not depend on temperature.

$L_{1}$ and $L_{0}$ being the SPW attenuation lengths corresponding respectively to $p=1$ and 0 , one obtains from (16a) and (16b) :

$$
L_{1} \# L_{\mathrm{cl}} \# \frac{\lambda_{0}^{2} Z_{0}}{2 \pi^{2}} \sigma
$$


and :

$$
\frac{1}{L_{0}} \# \frac{1}{L_{\mathrm{cl}}}\left[1+\frac{3}{8} k_{p}\left(\frac{\sigma}{l}\right)^{-1} \sigma\right]
$$

where $k_{p}=\omega_{p} / c$.

(17) can be written in the following interesting form :

$$
\frac{L_{\mathrm{cl}}}{L_{0}}-1 \# m_{0} \sigma
$$

with :

$$
m_{0}=\frac{3}{8} k_{p}\left(\frac{\sigma}{l}\right)^{-1} .
$$

Therefore, the curve $\mathcal{L}(\sigma)$ defined by :

$$
\mathfrak{L}(\sigma)=\frac{L_{\mathrm{cl}}}{L}-1
$$

should be very close to a straight line for $p=0$, the slope of which is the above characteristic constant $m_{0}$ of the metal.

As a matter of fact, the exact curve $\mathfrak{L}(\sigma)_{p=0}$ plotted on figure 6 is not very different from $m_{0} \sigma$.

At $\lambda=10 \mu \mathrm{m}$, the divergence between both curves is about $3 \%$ for all 6 .

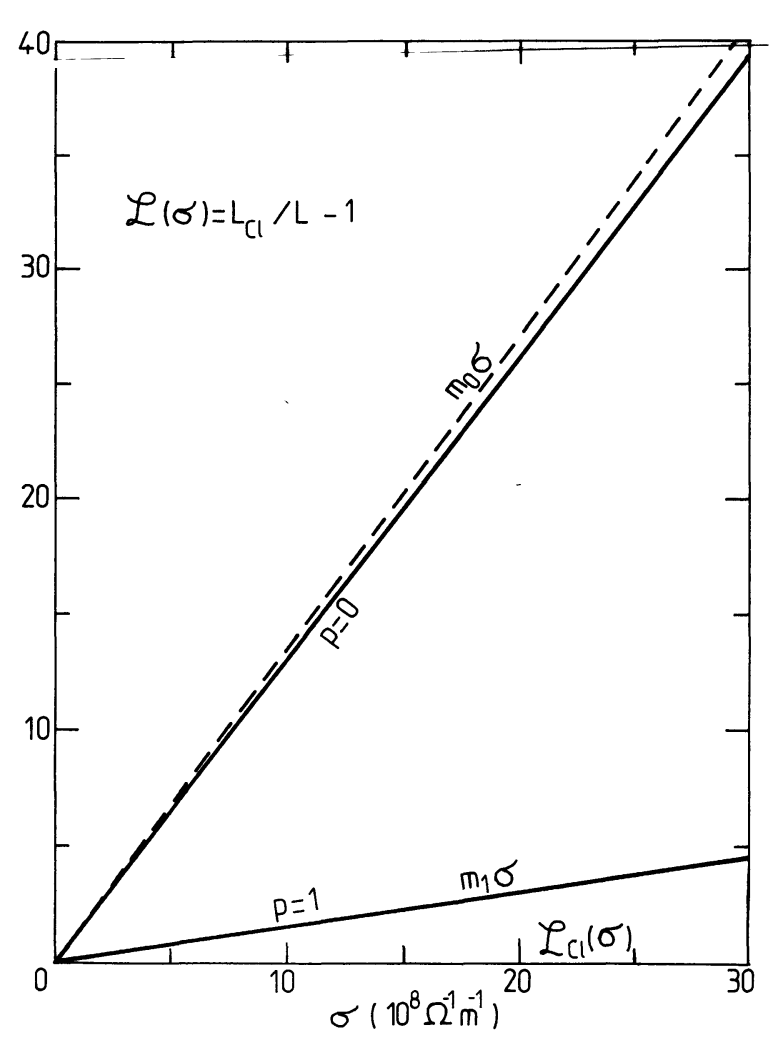

Fig. 6. - Curves $\mathcal{L}(\sigma)$ (see definition in text) obtained from exact theoretical calculations (continuous lines) and from the approximate expression (18) for $p=0$ (dashed line) at $\lambda=10 \mu \mathrm{m}$ for a Cu-vacuum interface.
The agreement should be better at shorter wavelengths and one may conclude that expressions (16b), (17) and (18) are good approximations in the diffuse scattering case.

In the specular scattering case, the approximate curve given by relation (16a) should be $\mathcal{L}_{\mathrm{cl}}(\sigma)$ that is the horizontal axis on figure 6.

Obviously, the exact curve $\mathfrak{L}(\sigma)_{p=1}$ and $\mathfrak{L}_{\mathrm{cl}}(\sigma)$ are not in good agreement.

Nevertheless, $\mathscr{L}(\sigma)_{p=1}$ is practically a straight line and that constitutes a heuristic argument according to which $\mathfrak{L}(\sigma)$ remains a straight line for a given $p$ and can be expressed by :

$$
\mathfrak{L}(\sigma)_{p}=m_{p} \sigma .
$$

If the slope $m_{p}$ is supposed to vary linearly with $p$ between $m_{0}$ and $m_{1}$, it will be given by the relation :

$$
m_{p} \approx(1-p) m_{0}+p m_{1} .
$$

Therefore, the experimental determination of $m$ could give an estimate of the scattering parameter :

$$
p \approx \frac{m_{0}-m_{p}}{m_{0}-m_{1}}
$$

If $m_{1}=0$, the usual heuristic attenuation relation given by other authors [10] is obtained :

$$
A=\frac{1}{L} \approx \frac{1}{L_{\mathrm{cl}}}\left(1+m_{p} \sigma\right)
$$

where :

$$
m_{p} \approx \frac{3}{8}(1-p) k_{p}\left(\frac{\sigma}{l}\right)^{-1}
$$

For copper, it is found :

$$
\begin{aligned}
& m_{1} \approx 0.16 \times 10^{-8} \Omega \mathrm{m} \\
& m_{0} \approx 1.36 \times 10^{-8} \Omega \mathrm{m}
\end{aligned}
$$

at $\lambda=10 \mu \mathrm{m}$.

If $\lambda$ decreases, $m_{1}$ decreases too, whereas $m_{0}$ remains a constant.

For very large conductivities, if $p$ is supposed to be independent on temperature, the attenuation length takes the asymptotic value given by :

$$
L(\sigma \rightarrow \infty)=\frac{L_{\mathrm{cl}}}{m_{p} \sigma}=\frac{1}{m_{p}} \frac{\lambda_{0}^{2} Z_{0}}{2 \pi^{2}} .
$$

The values computed with the above formula for the Cu-vacuum interface : $L_{0}(\infty) \approx 0.14 \mathrm{~m}$ and $L_{1}(\infty) \approx$ $1.20 \mathrm{~m}$, are in good agreement with the exact theoretical curves of figure 6 .

In the general case, if $p$ depends on the temperature, the curves $\mathfrak{L}(\sigma)$ will not be straight lines and their shape could give useful information on electronsurface phonon scattering. 


\section{Conclusion.}

The exact theoretical values of SPW attenuation have been computed for a $\mathrm{Cu}$-vacuum interface in the two limiting cases $p=0$ and 1 and this calculation led us to the following conclusions :

1. The attenuation length of the SPW is very sensitive to the scattering process of the conduction electrons at the interface for wavelengths in the domain $2<\lambda<10 \mu \mathrm{m}$.

2. When the scattering is completely diffuse $(p=0)$, the approximate dispersion relation (15b) gives accurate numerical results at wavelengths in the $2-10 \mu \mathrm{m}$ range for temperature ranging from a few $K$ up to room temperature.

3. When the scattering is completely specular $(p=1)$, the approximate dispersion relation (15a), identical to the result of the classical theory, diverges significantly from the exact one.

The usual heuristic expression (20) of the attenuation could therefore be improved if the slope factor $m$ is expressed by expression (19).

One could then estimate the scattering parameter $p$ from the experimental curve :

$$
\mathfrak{L}(\sigma)=\frac{L_{\mathrm{cl}}(\sigma)}{L_{\text {exp }}(\sigma)}-1
$$

$\sigma$ being the measured bulk conductivity, and the two limiting straight lines $m_{0} \sigma$ and $m_{1} \sigma$ (Fig. 6). Different values of $\sigma$ can be obtained by varying the temperature.

$m_{0}$ and $m_{1}$ are theoretical slopes computed from two characteristic constants of the metal, for example $v_{\mathrm{F}}$ and $\sigma / l$, or $v_{\mathrm{F}}$ and $\omega_{p}$.

One must take care to use the plasma angular frequency $\omega_{p}$ given by the formula $\left(N e^{2} / \varepsilon_{0} m^{*}\right)^{1 / 2}$ and not by $\left(N e^{2} / \eta_{\mathrm{r}} \varepsilon_{0} m^{*}\right)^{1 / 2}, \eta_{\mathrm{r}}$ being the relative dielectric constant of the metallic ions plus vacuum.

The latter is solution of the equation $\operatorname{Re}\left\{\eta_{\mathrm{m}}\right\}=0$ if $\operatorname{Im}\left\{\eta_{\mathrm{m}}\right\} \ll 1, \eta_{\mathrm{m}}$ being the classical Drude dielectric constant of the metal.

Moreover, the determination of $p$ described here is based on a linear interpolation of $m$ between $m_{1}$ and $m_{0}$ and there does not exist to our knowledge an exact theory allowing us to check the accuracy of the heuristic expression (19) for any $p$. (That will be the purpose of another publication, recent theoretical numerical results obtained by the author indicate that (19) is well verified in the near infrared in a large domain of temperature).

\section{References}

[1] Schulz, L. G., Adv. Phys. 6 (1957) 102.

[2] Reuter, G. E. H., Sondheimer, E. H., Proc. R. Soc. A 195 (1948) 336

[3] Dingle, R. B., Physica XIX (1953) 348.

[4] Fuchs, K., Proc. Cambridge Philos. Soc. 34 (1938) 100.

[5] Kliewer, K. L., Fuchs, R., Phys. Rev. 172 (1968) 607.

[6] Jones, W. E., Kliewer, K. L., Fuchs, R., Phys. Rev. 178 (1969) 1201.

[7] Saermark, K., J. Phys. F 10 (1980) 2447.

[8] Mattis, D. C., Dresselhaus, G., Phys. Rev. 111 (1958) 403.

[9] Lenham, A. P., Treherne, D. M., J. Opt. Soc. Am. 56 (1966) 683.

[10] Schlesinger, Z., Sievers, A. J., Solid State Commun. 43 (1982) 671.

[11] McKay, J. A., Rayne, J. A., Phys. Rev. B 13 (1976) 673.

[12] Nguyen Van V., Gandais, M., Couzineau, M. M., Congrès International sur les couches minces, Cannes (1970).

[13] Elsom, K. C., Sambles, J. R., J. Phys. F 11 (1981) 647.

[14] Soffer, S. B., J. Appl. Phys. 38 (1967) 1710.
[15] Schoenwald, J., Burstein, E., Elson, J. M., Solid State Commun. 12 (1973) 185.

[16] Schoenwald, J., Burstein, E., Proc. Taormina Conf. on Polaritons, Edited by E. Burstein (Pergamon N.Y.) 1974 , p. 139.

[17] McMullen, J. D., Solid State Commun. 17 (1975) 331.

[18] Dingle, R. B., Physica XIX (1953) 311.

[19] Burstein, E., et al., Proc. of the 1st Taormina Res. Conf. on the structure of matter 89 (1972).

[20] Lopez-Rios, T., Doctor Thesis, Paris VI (1980).

[21] Melnyk, A. R., Harrison, M. J., Phys. Rev. B 2 (1970) 835.

[22] Keller, J. M., Fuchs, R., Kliewer, K. L., Phys. Rev. B 12 (1975) 2012.

[23] Dingle, R. B., Appl. Sci. Res. B 3 (1953) 69.

[24] Peterseim, J., Thummes, G., Mende, H. H., Phys. Status Solidi 59 (1980) K25.

[25] Dujardin, M. M., Theye, M. L., J. Phys. Chem. Solids 32 (1971) 2033.

[26] KlIEWER, K. L., Surface Science 101 (1980) 57. 